\title{
SDSS 0956+5128: A BROAD-LINE QUASAR WITH EXTREME VELOCITY OFFSETS
}

\author{
Charles L. Steinhardt ${ }^{1}$, Malte Schramm ${ }^{1}$, John D. Silverman ${ }^{1}$, Rachael Alexandroff ${ }^{2}$, Peter CapaK $^{3}$, \\ Francesca Civano $^{4}$, Martin Elvis $^{4}$, Dan Masters $^{5,6}$, Bahram Mobasher ${ }^{5}$, \\ Petchara Pattarakijwanich ${ }^{2}$, And Michael A. Strauss ${ }^{2}$ \\ ${ }^{1}$ Kavli IPMU, University of Tokyo, Kashiwanoha 5-1-5, Kashiwa-shi, Chiba, Japan \\ ${ }^{2}$ Department of Astrophysical Sciences, Princeton University, Ivy Lane, Princeton, NJ 08544, USA \\ ${ }^{3}$ California Institute of Technology, MC 249-17, 1200 East California Boulevard, Pasadena, CA 91125, USA \\ ${ }^{4}$ Harvard-Smithsonian Center for Astrophysics, 60 Garden St., Cambridge, MA 02138, USA \\ ${ }^{5}$ Department of Physics and Astronomy, University of California, Riverside, CA 92508, USA \\ ${ }^{6}$ Observatories of the Carnegie Institution of Washington, Pasadena, CA 91101, USA \\ Received 2012 June 20; accepted 2012 September 4; published 2012 October 12
}

\begin{abstract}
We report on the discovery of a Type 1 quasar, SDSS $0956+5128$, with a surprising combination of extreme velocity offsets. SDSS $0956+5128$ is a broad-lined quasar exhibiting emission lines at three substantially different redshifts: a systemic redshift of $z \sim 0.714$ based on narrow emission lines, a broad Mg II emission line centered $1200 \mathrm{~km} \mathrm{~s}^{-1}$ bluer than the systemic velocity, at $z \sim 0.707$, and broad $\mathrm{H} \alpha$ and $\mathrm{H} \beta$ emission lines centered at $z \sim 0.690$. The Balmer line peaks are $4100 \mathrm{~km} \mathrm{~s}^{-1}$ bluer than the systemic redshift. There are no previously known objects with such an extreme difference between broad Mg II and broad Balmer emission. The two most promising explanations are either an extreme disk emitter or a high-velocity black hole recoil. However, neither explanation appears able to explain all of the observed features of SDSS $0956+5128$, so the object may provide a challenge to our general understanding of quasar physics.
\end{abstract}

Key words: accretion, accretion disks - black hole physics - galaxies: evolution - galaxies: nuclei - quasars: general

Online-only material: color figures

\section{INTRODUCTION}

The broad emission lines distinctive of quasar spectra are believed to come from high-velocity gas in the "broad-line region" (BLR), $\sim 0.1-1 \mathrm{pc}$ from the central engine, with typical full width at half-maximum (FWHM) in the range of 2000-20,000 km s${ }^{-1}$ (see Peterson 2008). Prominent broad emission lines visible in optical spectra at $z \sim 0.7$ include $\mathrm{H} \beta$ and $\mathrm{Mg}$ II, with $\mathrm{H} \alpha$ visible in the near-infrared and $\mathrm{CIV}$ in the UV. Quasar broad emission lines are usually assumed to be predominantly virial and are typically symmetric (Murray et al. 1995). Indeed, black hole mass estimators have been developed predicated upon this virial assumption and Gaussian line profiles (McLure \& Jarvis 2002; McLure \& Dunlop 2004; Vestergaard 2002; Vestergaard \& Peterson 2006; Wang et al. 2009; Onken $\&$ Kollmeier 2008; Shen et al. 2008; Risaliti et al. 2009; Rafiee \& Hall 2011).

However, there are many known sources of non-virial motion, often leading to asymmetric broad-line profiles that may have peaks offset from the systemic redshift of the host. Strong inflows or outflows would shift the peak of BLR lines. Similarly, radiation pressure and/or quasar winds can yield an asymmetric C Iv line (Marconi et al. 2009; Marziani et al. 2010), as can broad absorption lines. Typical asymmetries are several hundreds to $2000 \mathrm{~km} \mathrm{~s}^{-1}$.

A much larger offset in the broad emission line peaks may be caused by eccentric disk emission. Emission lines originating in a relativistic, eccentric disk may have very asymmetric profiles with double peaks (Chen \& Halpern 1989; Eracleous et al. 1995). Numerous examples of these disk or double-peaked emitters have been found in the Sloan Digital Sky Survey (SDSS) quasar catalog (Strateva et al. 2003), typically based upon $\mathrm{H} \beta$ and $\mathrm{H} \alpha$ profiles and $\mathrm{Mg}$ II for objects at higher redshift (Luo et al. 2009). The blue peak of these Balmer lines is often offset by several thousand $\mathrm{km} \mathrm{s}^{-1}$ from the wavelength corresponding to the systemic narrow-line redshift (Strateva 2004). If the asymmetry from Doppler boosting is strong enough, there may appear to be only one, blueshifted peak in a skewed line profile (e.g., Eracleous et al. 1995, Figure 4(c)).

The post-merger recoil of central supermassive black holes (SMBHs) can also give rise to offset broad emission-line peaks. If each galaxy involved in a merger has already formed an SMBH, dynamical friction brings the two black holes into a black hole binary (Yu \& Tremaine 2002; Di Matteo et al. 2005; Escala et al. 2004; Merritt \& Milosavljević 2005; Mayer et al. 2007; Dotti et al. 2006; Colpi \& Dotti 2009), from which the binary pair can eventually coalesce into one SMBH (see Pretorius 2007). Numerical simulations reveal a final phase in which the emission of gravitational radiation produces a spin-dependent recoil of the resulting black hole, ranging from $\lesssim 200 \mathrm{~km} \mathrm{~s}^{-1}$ for non-spinning black holes to perhaps $\gtrsim 1000 \mathrm{~km} \mathrm{~s}^{-1}$ for spinning black holes in special orientations (Campanelli et al. 2007; Herrmann et al. 2007; Schnittman \& Buonanno 2007; Loeb 2007). Observational signatures of such a recoil might include a quasar BLR moving at $\gtrsim 1000 \mathrm{~km} \mathrm{~s}^{-1}$ relative to its host galaxy and the quasar narrow lines (Loeb 2007).

There are no known, confirmed examples of recoiling SMBHs. Several examples of binary black holes have been found at low redshift $(z<0.05$; Komossa et al. 2003; Ballo et al. 2004; Hudson et al. 2006; Rodriguez et al. 2006; Bianchi et al. 2008; Comerford et al. 2009; Liu et al. 2010; McGurk et al. 2011; Max et al. 2007; Barrows et al. 2012), with separations in the kiloparsec range. Attempts to systematically search for recoiling black hole candidates in which optical spectra show multiple line systems at slightly different redshifts (Bonning et al. 2007; Boroson \& Lauer 2009; Jahnke et al. 2009; 
Table 1

Emission Line Properties of SDSS 0956+5128

\begin{tabular}{lccc}
\hline \hline Line & $z$ & Observations & Clear Asymmetry \\
\hline Mg II $\lambda 2798$ & 0.707 & DR3, SEGUE-2 & No \\
{$\left[\mathrm{O}_{\text {II }}\right] \lambda 3727$} & 0.714 & DR3, SEGUE-2 & No \\
$\mathrm{H} \beta \lambda 4861$ & 0.690 & DR3, SEGUE-2, DEIMOS & Yes \\
{$\left[\mathrm{O}_{\text {III }}\right] \lambda 4959$} & 0.714 & DR3, SEGUE-2, DEIMOS & No \\
{$\left[\mathrm{O}_{\text {III }}\right] \lambda 5007$} & 0.714 & DR3, SEGUE-2, DEIMOS & No \\
$\mathrm{H} \alpha \lambda 6563$ & 0.690 & TripleSpec & Yes \\
\hline
\end{tabular}

Liu et al. 2010; Decarli et al. 2010; Tsalmantza et al. 2011) have yielded several candidates (Komossa et al. 2008), but there is typically a more mundane, alternative explanation as well (Shields et al. 2009). In particular, HE 0450-2958 (Magain et al. 2005; Hoffman \& Loeb 2006) was first claimed to have no host galaxy and thus be a good candidate for an ejected black hole, but it may have a faint host galaxy after all, and even shows signs of ongoing star formation, while it does not show the necessary offset between narrow and broad emission lines that must be characteristic of a recoiling black hole (Merritt et al. 2006; Kim et al. 2007; Jahnke et al. 2009). Most recently, CID-42 (Comerford et al. 2009; Civano et al. 2010), at $z=$ 0.359 , includes two optical sources offset by $1200 \mathrm{~km} \mathrm{~s}^{-1}$ lying $2.4 \mathrm{kpc}$ apart in the same galaxy, and appears to be a good candidate for either a recoiling black hole or a system containing two active galactic nuclei (AGNs), one Type 1 and the other Type 2. New observations using Chandra appear to rule out the latter scenario (Civano et al. 2012).

In this work, we describe SDSS J095632.49+512823.92 (hereafter SDSS 0956+5128), a broad-lined Type 1 quasar exhibiting lines with three substantially different redshifts: a systemic redshift for quasar narrow emission lines, a second, $1200 \mathrm{~km} \mathrm{~s}^{-1}$ bluer, associated with broad Mg II emission, and a third, $4100 \mathrm{~km} \mathrm{~s}^{-1}$ bluer than the narrow lines, for broad $\mathrm{H} \alpha$ and $\mathrm{H} \beta$ (Table 1). This combination of spectral lines is very difficult to understand. Explanations for a blueshifted BLR relative to the host include (1) an undetected host galaxy lying at a lower redshift along the same line of sight, (2) a disk or doublepeaked emitter (Strateva et al. 2003), (3) a multiple-black-hole system in the host galaxy, or (4) a recoiling black hole. However, we know of no explanation for a Type I quasar in which one broad line is blueshifted by $2900 \mathrm{~km} \mathrm{~s}^{-1}$ relative to the other. No other systems with three such velocities were found in a search of the SDSS DR8 (Aihara et al. 2011) quasar catalog. Therefore, SDSS 0956+5128 appears to be a rare object, and thus might be difficult to fit into existing models because it may be a relatively short-lived state. If so, SDSS 0956+5128 may reveal a previously unobserved phase in the SMBH life cycle.

In Section 2, we describe SDSS observations of SDSS 0956+5128. Section 2.1 describes follow-up spectroscopic observations of $\mathrm{H} \alpha, \mathrm{H} \beta$, and $\mathrm{Mg}$ II 4-5 years (in the rest frame) after the initial SDSS spectrum, confirming that there are indeed three different velocities in this system. Subaru Infrared Camera and Spectrograph (IRCS) $J$-, $H$-, and $K$-band images of the host galaxy, in addition to GALEX and Two Micron All Sky Survey (2MASS) observations, enable us to address whether a recent merger has occurred (Section 3). Finally, in Section 4 we consider the two models that might be most capable of producing such a system. We discuss whether SDSS 0956+5128 might be an extreme disk emitter with unique features. Alternatively, we consider the possibility that SDSS $0956+5128$ might be a recoiling post-merger SMBH. Neither appears to be a good explanation for the three observed velocities in SDSS 0956+5128.
Table 2

Summary of Observations of SDSS 0956+5128

\begin{tabular}{lccr}
\hline \hline Instrument/Survey & MJD & Wavelengths & $\begin{array}{c}\text { Exposure Time } \\
(\mathrm{s})\end{array}$ \\
\hline SDSS plate 902 & 52409 & $3900-9200 \AA$ & 2700 \\
SEGUE-2 plate 3320 & 54912 & $3900-9200 \AA$ & 900 \\
DEIMOS & $55888-55889$ & $6500-9000 \AA$ & 2400 \\
TripleSpec & 55922 & $9500-24600 \AA$ & 1920 \\
Subaru IRCS & 55972 & $H$-band & 2736 \\
Subaru IRCS & 55972 & $J$-band & 3168 \\
Subaru IRCS & 55972 & $K$-band & 3195 \\
Subaru IRCS & 55984 & $K$-band & 2910 \\
Subaru IRCS & 55985 & $K$-band & 2500 \\
\hline
\end{tabular}

\section{SDSS 0956+5128 IN SDSS}

In a 2002 DR3 spectrum (Abazajian et al. 2005), SDSS 0956+5128 (Figure 2) clearly shows a broad-lined Type I quasar, selected initially based upon its colors in five filters (Richards et al. 2002) for spectroscopic follow-up.

The SDSS redshift of $z=0.714$ is a good fit for the narrow emission lines, including [O II] $\lambda 3727$, [O III] $\lambda 4959,5007$, and the narrow component of $\mathrm{H} \beta$ (Figure 1). The broad emission lines, however, lie at lower velocities. We fit the continuum, Fe II emission, and the broad Mg II line following McLure \& Dunlop (2004) and Shen et al. (2008). The best-fit Mg II line has a redshift $0.7071 \pm 0.0006$ and FWHM $12800 \pm 490 \mathrm{~km} \mathrm{~s}^{-1}$, with the $11 \sigma$ difference providing compelling evidence for a BLR offset velocity of $1200 \mathrm{~km} \mathrm{~s}^{-1}$ from the narrow lines. The $z=0.7071$ fit has a $\chi^{2} /$ dof of 0.97 over wavelengths within $10,900 \mathrm{~km} \mathrm{~s}^{-1}(2 \sigma)$ of the best-fit line centroid, compared to 1.36 for the best $z=0.714$ fit.

The broad component of $\mathrm{H} \beta$ is also blueshifted relative to $z=0.714$. The centroid of the broad component is at $z=0.690$, apparently blueshifted by approximately $4100 \mathrm{~km} \mathrm{~s}^{-1}$ relative to the narrow lines, compared to $1200 \mathrm{~km} \mathrm{~s}^{-1}$ for $\mathrm{Mg}$ II. We also note that $\mathrm{Mg}$ II is symmetric, while $\mathrm{H} \beta$ is not, even though they have similar ionization potentials and should be emitted from gas clouds at a similar radius from the central black hole (see McLure \& Jarvis 2002).

\subsection{Confirming Observations of Broad Emission Line Velocity Offsets With Optical Spectra}

The offset between the broad $\mathrm{H} \beta$ and $\mathrm{Mg}$ II emission lines is sufficiently surprising and difficult to explain that we must consider whether measurement error, fitting error, or absorption from the host galaxy might be responsible for mislocating one of the line centroids. SDSS 0956+5128 was observed a second time using the SDSS spectrographs as part of SEGUE-2 (Yanny et al. 2009; Figures 1 and 2), seven years after the initial spectrum (it was erroneously targeted as a candidate blue horizontal-branch star). The SEGUE-2 spectrum has higher signal to noise and is consistent with the previous SDSS measurement of $\mathrm{Mg}$ II at $z=0.707, \mathrm{H} \beta$ at $z=0.690$, and the narrow lines at $z=0.714$.

Because the $\mathrm{H} \beta$ profile is asymmetric and has such a large offset, we performed two additional follow-up observations (Table 2). Using Keck/DEIMOS (Faber et al. 2003), a higherresolution and higher signal-to-noise spectrum using the $830 \mathrm{G}$ grating ( $R \sim 3200$, compared to $\sim 2000$ for SDSS) was taken on 2011 November 23-24, under photometric conditions and 0.6-0.'8 seeing. On November 23 two 600 s exposures were taken at a slit position angle (P.A.) of 90 and on November 24 $900 \mathrm{~s}$ and $300 \mathrm{~s}$ exposures were taken at a slit P.A. of 0. 


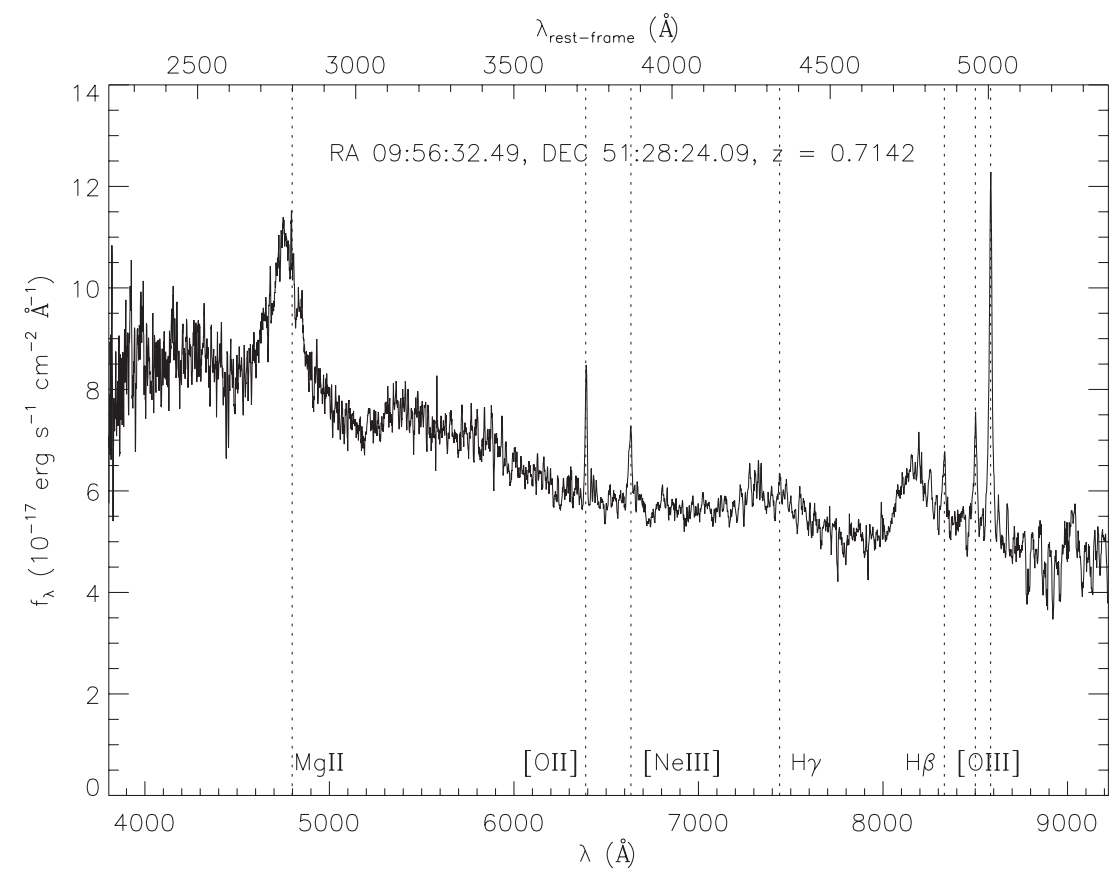

Figure 1. Spectrum of SDSS $0956+5128$ from SEGUE-2. The identified redshift of $z=0.714$ is a good fit to narrow emission lines such as [O II], [O III], and the narrow component of $\mathrm{H} \beta$. However, the broad emission lines $\mathrm{H} \beta$ and $\mathrm{Mg}$ II are offset by $4100 \mathrm{~km} \mathrm{~s}^{-1}$ and $1200 \mathrm{~km} \mathrm{~s}^{-1}$, respectively.
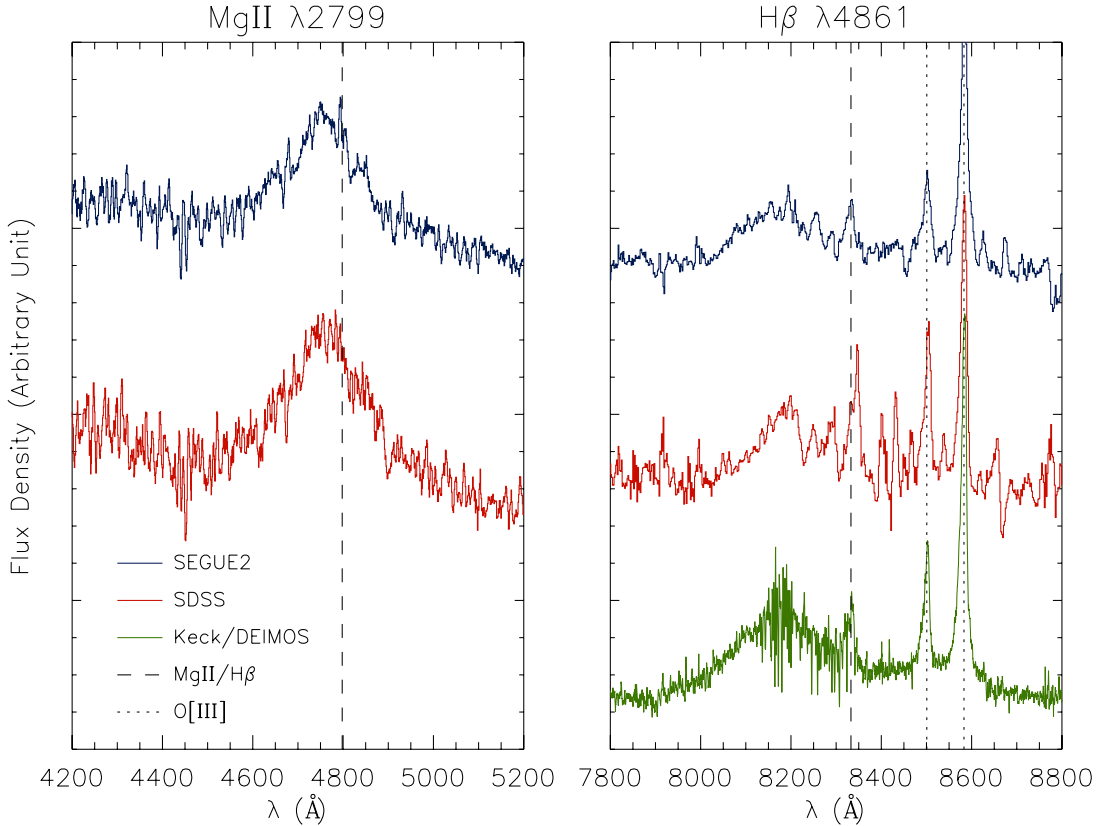

Figure 2. Comparison of $\mathrm{Mg}$ II and $\mathrm{H} \beta$ as observed by SEGUE-2 (dark blue), Keck/DEIMOS (green), and in the original SDSS spectrum (red). The Keck/DEIMOS spectrum has the highest signal to noise, followed by SEGUE-2 and then SDSS DR3. The expected wavelength given the redshift of the systemic narrow lines is indicated (dashed). The broad emission line centroids lie at similar velocities in all spectra, and the spectra are consistent with being identical.

(A color version of this figure is available in the online journal.)

The $1200 \mathrm{G}$ grating tilted to $7800 \AA$ was used with a OG550 blocking filter and 0.5 slit, resulting in a spectral resolution of $0.43 \AA$ FWHM measured from sky lines. The data were reduced with a modified version of the DEIMOS reduction package which accounts for guider drift, variable seeing, wavelength calibration errors, and non-photometric conditions.

The spectro-photometric standard star HZ-44 was used for relative flux calibration. Regions of telluric absorption and strong spectral lines in HZ-44 were masked, and then a 10th order polynomial was fit to the ratio of measured to expected flux. After scaling out the baseline throughput, Gaussian lines were fit to the residual flux ratio at the location of known telluric absorption lines to account for atmospheric absorption. Both spectra show $\mathrm{H} \beta$ in the same location as the SDSS spectra (Figure 2). The $\mathrm{H} \beta$ profile shows similar asymmetry in both SDSS and Keck spectra.

\subsection{Near-infrared Spectroscopy}

In addition, an infrared spectrum was taken using TripleSpec (Wilson et al. 2004; Rayner et al. 2003; Cushing et al. 2004; Vacca et al. 2003; $R \sim 2800$ ) for eight $240 \mathrm{~s}$ exposures in 2011 December on the Astrophysical Research Consortium $3.5 \mathrm{~m}$ at Apache Point Observatory. This spectrum reveals a broad 


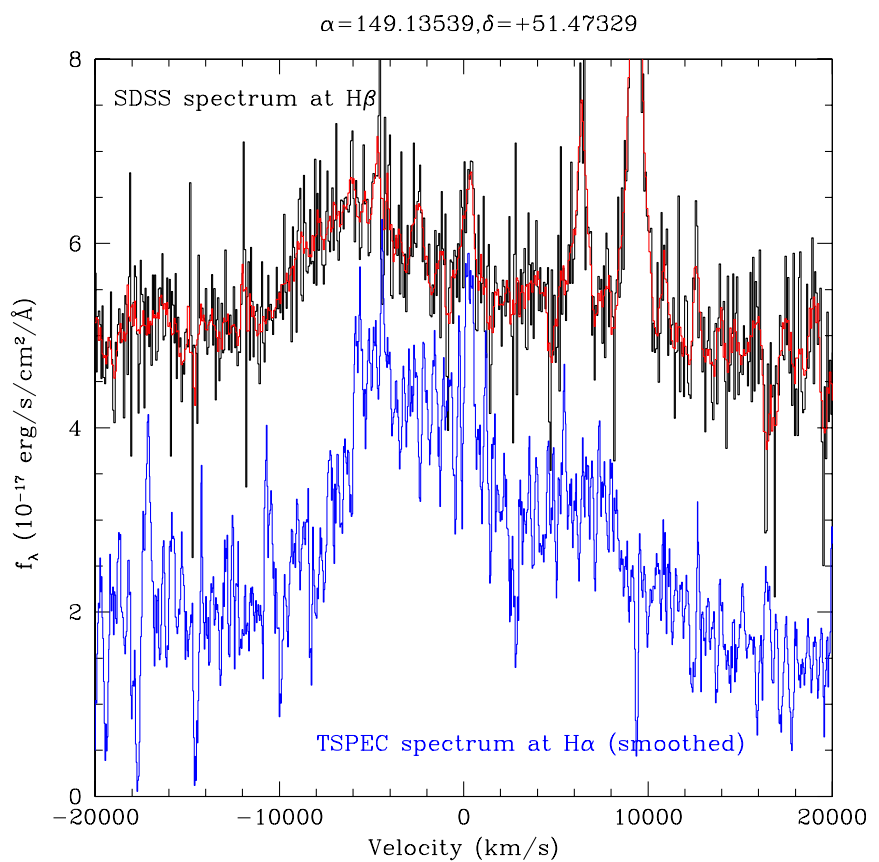

Figure 3. Comparison of $\mathrm{H} \alpha$ as observed by TripleSpec on the ARC $3.5 \mathrm{~m}$ and $\mathrm{H} \beta$, as observed by SEGUE- 2 . The broad and narrow components are similarly offset and the lines have similar overall profiles. Velocities are indicated relative to the best-fit $z \sim 0.714$ for the narrow emission lines. The apparent absorption feature at $\sim 2500 \mathrm{~km} \mathrm{~s}^{-1}$ in $\mathrm{H} \alpha$ coincides with atmospheric absorption lines. While the TripleSpec spectrum has been corrected for atmospheric absorption from observations of a nearby A star, this feature may be a residual rather than absorption associated with the quasar or its host.

(A color version of this figure is available in the online journal.)

$\mathrm{H} \alpha$ emission line at $z=0.690$ with a profile comparable to $\mathrm{H} \beta$ (Figure 3), with no evidence of strong absorption lines in either line. We note that although the strong asymmetry in $\mathrm{H} \alpha$ and $\mathrm{H} \beta$ is one characteristic of disk or double-peaked emitters (Eracleous et al. 1995; Strateva et al. 2003), as above, the large offset between $\mathrm{H} \beta$ and $\mathrm{Mg}$ II is not characteristic of the few examples with well-measured $\mathrm{H} \beta$ and $\mathrm{Mg}$ II. We conclude that the measured centroids for the Balmer lines indeed lie at a different redshift than the narrow lines. In Table 2, we summarize the observations, both spectroscopy and imaging, of SDSS $0956+5128$ that we use in this study.

\section{THE HOST GALAXY}

A key question in understanding SDSS 0956+5128 lies in determining whether there are multiple galaxies along the same line of sight. If there were multiple systems, at least the offset between broad emission lines and narrower galactic lines would be easily explained. However, if SDSS 0956+5128 is indeed one system with three distinct velocities, it would be more puzzling. In particular, if the peculiar features of SDSS 0956+5128 are due to a black hole recoil, we can make several predictions about its host. Most importantly, the black hole should indeed be associated with the $z \sim 0.714$ galactic counterpart. Attenuation of the quasar spectral energy distribution (SED) due to a dusty host that recently underwent a merger could be evidence that we are investigating one system, not two.

\subsection{Broadband SED}

We consider the broadband SED of the source combining SDSS data with GALEX (Martin et al. 2005), WISE (Wright et al. 2010), and 2MASS (Skrutskie et al. 2006). We find that the

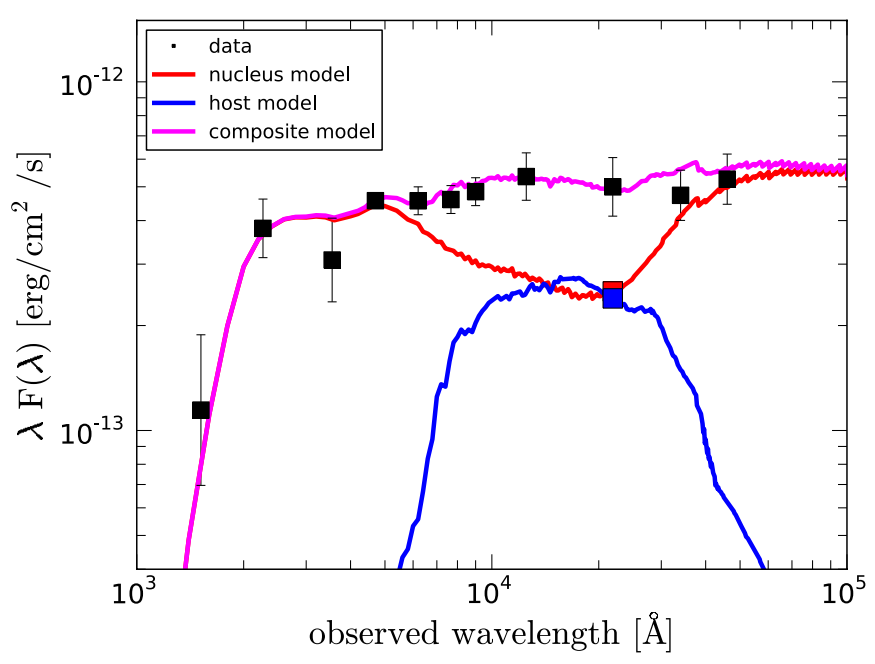

Figure 4. Best-fit decomposition of the observed SDSS 0956+5128 SED into an active nucleus (red) and host galaxy (blue). The sum of the two components (magenta) is matched to the observed SED (black).

(A color version of this figure is available in the online journal.)

optical data require $A_{\mathrm{V}}=0.3$ reddening of the SDSS composite quasar spectrum (Vanden Berk et al. 2001; Richards et al. 2006). Even this is not enough to reproduce the observed level of IR emission. Therefore, we have added a galaxy spectrum with primarily an old stellar population from Maraston (2005). We make use of the $K$-band (Section 3.2) image decomposition to constrain the AGN template and the galaxy template in our SED fit. This is important to reduce the degeneracies between the different models. This decomposition is shown in Figure 4. We note that some photometric data points are likely to be affected by strong emission lines (i.e., $\operatorname{Ly} \alpha, \mathrm{H} \alpha$ ). Both the quasar and inferred host galaxy appear to be dusty.

From our decomposition, we roughly estimate the stellar mass of the galaxy to be $\sim 8 \times 10^{10} M_{\odot}$. We also use our $\mathrm{Mg}$ II and the strength of the nearby continuum fit to infer a central black hole mass of $\log \left(M / M_{\odot}\right)=8.65$ using the $\mathrm{Mg}$ II-based virial mass estimator calibrated by McLure \& Dunlop (2004). This estimate may be incorrect if there is substantial non-virial motion, although the Mg II line appears symmetric despite its offset. The estimated black hole mass and galaxy luminosity are consistent with a central black hole-galaxy pair lying near the $z=0 M_{\mathrm{BH}}-L_{\text {gal }}$ relation (Bentz et al. 2009). However, our inferred galaxy is likely a factor of $\sim 4$ lower mass than necessary to lie on the $M_{\mathrm{BH}}-M_{\text {bulge }}$ relation (Häring \& Rix 2004). Because the inferred host lies either on or below these relations, any different counterpart corresponding to the quasar should be nearly as bright as our inferred host.

\subsection{High-resolution IR Imaging with Subaru}

Stronger evidence that the quasar lies in the inferred host comes from follow-up $J$-, $H$-, and $K$-band images using the Subaru IRCS with adaptive optics (AO; Hayano et al. 2008, 2010). We performed this imaging using the AO188 Adaptive Optics system in laser guide star (LGS) mode, with the LGS equidistant from the quasar and point-spread function (PSF) star in order to improve PSF stability. The $K$-band image with 52 mas pixels shown in Figure 5 was taken with a net exposure time of 66 minutes but varying conditions. The PSF was then modeled using a star of brightness similar to the main quasar point source, as a combination of a Gaussian core and Moffat 


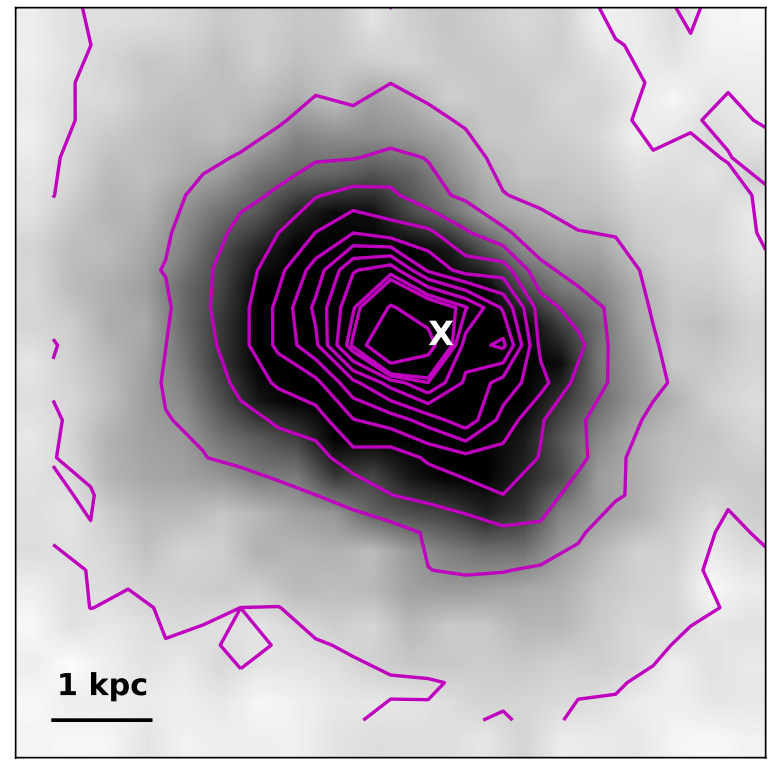

Figure 5. Infrared emission of the host galaxy of SDSS 0956+5128 as observed by the Subaru IRCS ( $K$-band), with a resolution of 0.15 arcsec. The quasar emission, with its centroid marked in white, has been subtracted. The quasar point source appears to lie away from the center of an asymmetric galaxy, which may indicate recent merger activity. Contours are marked on a linear scale.

(A color version of this figure is available in the online journal.)

wing, and rotated to correct for mismatched alignment between the source, LGS, and PSF star.

Only the $K$-band image shown in Figure 5, with a PSF of 0.15 arcsec, was suitable for our purposes. The second $K$-band image was taken with a 20 mas pixel scale. Therefore, the flux per pixel is much lower and our integration time was too short. We did not reach the necessary depth to determine properties of the host, although extended host emission can be detected. The $J, H, K p$ images were taken in service mode with a P.A. $=90$. This leads to different distances from the guide star to the PSF and the QSO. Anisoplanatism degrades the seeing and cannot be controlled with effectively one PSF star. So, we cannot predict the shape of the PSF at the quasar position. Therefore, we decided to reject those images in the analysis.

We then decompose the $K$-band image into a point source and host using GALFIT v. 3.0 (Peng et al. 2010). First a PSFonly model is fit to the data, then a Sersic profile is fit for the host galaxy contribution. We find that the AGN-host flux ratio is $1.0 \pm 0.4$, and the best-fit parameters for the host are those of a compact, early-type galaxy $\left(n_{\text {Sersic }} \sim 3.7-4.5, a / b=\right.$ $0.50 \pm 0.15, R_{1 / 2} \sim 1.3 \mathrm{kpc}$ ). The resulting picture is thus consistent with a point source lying in a single host galaxy (Figure 5).

There appear to be asymmetric structures in the central pixels, but due to the difficulties in controlling the AO PSF, we cannot be certain that they are real. Surprisingly, the quasar appears not to be located at the highest concentration of starlight, likely the galactic bulge. If SDSS $0956+5128$ does not include a recoiling black hole, this is difficult to understand even in the case of a merger, particularly coupled with the high velocity. However, the apparent offset may be an artifact caused by imperfections in the point-source subtraction.

\section{DISCUSSION}

SDSS 0956+5128 appears to be unique in the SDSS DR8 catalog in that there are emission lines at three velocities in the system, corresponding to $z \sim 0.714$ (narrow lines), $z \sim 0.707$
( $\mathrm{Mg}$ II), and $z \sim 0.690(\mathrm{H} \alpha$ and $\mathrm{H} \beta$ ). The combination of these lines is startling. We considered four possible explanations: (1) an undetected host galaxy lying at a lower redshift along the same line of sight, (2) a disk or double-peaked emitter (Strateva et al. 2003), (3) a multiple-black-hole system in the host galaxy, or (4) a recoiling black hole.

If there were two Type 1 quasars involved, there should be two $\mathrm{Mg}$ II and two $\mathrm{H} \beta$ broad lines, lying $2900 \mathrm{~km} \mathrm{~s}^{-1}$ apart. SDSS 0956+5128 exhibits just one broad emission line of each species, which would require two unusual quasars, one with very weak $\mathrm{Mg}$ II and another with very weak $\mathrm{H} \beta$, both lying at a different velocity from that of the narrow lines. Even if a third SMBH were responsible for the narrow lines, a triple system model has the same flaw, that each black hole should have a discernible set of lines, and SDSS $0956+5128$ only has one set of narrow lines, one $\mathrm{Mg}$ II line, and one set of Balmer lines.

Disk emitters have been observed with offsets of $4100 \mathrm{~km} \mathrm{~s}^{-1}$ or more between broad and narrow lines (Strateva et al. 2003), but none of the handful of disk emitters with spectra containing both $\mathrm{H} \beta$ and $\mathrm{Mg}$ II has a clear offset between $\mathrm{H} \beta$ and $\mathrm{Mg}$ II. Given their large velocity offsets, disk emitters are likely examples of extreme accretion physics, so perhaps SDSS $0956+5128$ is an even more extreme system than previous examples, producing sharply different line profiles for lines even of similar ionization potential. Similarly, a recoiling black hole could potentially have a large offset between various broad and narrow lines, but none has ever been observed. We describe the case for each interpretation below.

\subsection{Is SDSS 0956+5128 an Extreme Disk Emitter?}

The Balmer lines in this object are strongly skewed with a strong peak blueward of the systemic velocity. Such objects cannot be fit with a typical, circular disk, even with Doppler boosting. However, they can be fit by models such as an eccentric disk (Eracleous et al. 1995) in which an additional parameter is added.

Sequences of various line profiles with different parameters can be found in Eracleous et al. (1995) and Strateva (2004). A line profile with a strong blue peak $4000 \mathrm{~km} \mathrm{~s}^{-1}$ bluer than the central value and a low-amplitude red peak is predicted for models with high values of the disk orientation $\varphi_{0}$, high eccentricity, and a small inner pericenter distance $\xi_{1}$. We note that our ability to fit such a line shape using an eccentric disk may not be all that surprising due to the large number of parameters. The seven parameters (in addition to the central black hole mass) allowed by an eccentric disk, combined with noise and the difficulties of decomposing the spectrum into $\mathrm{H} \beta$ broad and narrow components and $\left[\mathrm{O}_{\mathrm{III}}\right] \lambda 4959,5007$, might mean such a model will fit almost any skewed line shape. Further, other sorts of non-circular disk may also be consistent with this line profile, i.e., such a fit is not unique.

Regardless, an eccentric disk model does not produce different line shapes for $\mathrm{Mg} \mathrm{II}$ and $\mathrm{H} \beta$, since the ionization potentials of the two species are similar. The parameters producing a strongly skewed $\mathrm{H} \alpha$ and $\mathrm{H} \beta$ will produce a similarly shaped Mg II line, also with a peak at $z \sim 0.690$ instead of $z \sim 0.707$. So, although the Balmer lines alone could be consistent with SDSS $0956+5128$ as a disk emitter, $\mathrm{Mg}$ II is inconsistent with this interpretation.

\subsection{Is SDSS 0956+5128 a Recoiling Black Hole?}

Merritt et al. (2006) proposed that a post-merger, recoiling black hole could produce a symmetric BLR, offset by 
$\gtrsim 1000 \mathrm{~km} \mathrm{~s}^{-1}$ from the narrow-line region velocity. Could SDSS $0956+5128$ be an example of such a recoil? We must show that the $\mathrm{SMBH}$ is associated with the galaxy emitting the narrow lines at $z \sim 0.714$. Because of the high redshift, we cannot rule out that the black hole lies in another galaxy along the line of sight. Rather, we must rely on indirect evidence, considering whether the observed galaxy is consistent with being the host of the SMBH responsible for Balmer and $\mathrm{Mg}$ II emission.

The Subaru IRCS imaging reveals only one host, indicating that if there are multiple galaxies along the same line of sight, most of the galactic flux is coming from one, largest galaxy. The observed galaxy is a promising recoil candidate in that a decomposition into a galactic and quasar SED shows extinction that could be characteristic of a dusty galaxy. Mergers would likely be associated with strong extinction, although galaxies not undergoing mergers can also be dusty at many stages of their evolution.

Moreover, the inferred host galaxy mass is $8 \times 10^{10} M_{\odot}$, with a luminosity close to that expected for a $10^{8.65} M_{\odot}$ black hole lying on the $M-L_{\text {gal }}$ relation. A $10^{8.65} M_{\odot}$ central black hole is unlikely to lie in a substantially less luminous galaxy than the one detected by SDSS, and therefore the BLR is unlikely to belong to a quasar with a different galactic counterpart.

The presence of broad emission lines is evidence that if this is a recoiling black hole, the recoil took place in the recent past. Using an $\alpha$-disk (Shakura \& Sunyaev 1973) model, the accretion disk can temporarily survive the recoil and the BLR will continue to emit over a lifetime of (Loeb 2007)

$$
t_{\text {disk }}=8.4 \times 10^{6} \alpha_{-1}^{-0.8} \eta^{0.4} M_{7}^{1.2} v_{8}^{-2.8} \mathrm{yr} .
$$

For a coalesced black hole mass of $10^{8.65} M_{\odot}\left(M_{7}=45\right)$ and speed of $v_{8}=1.21$, the dimensionless combination of radiative efficiency and Eddington ratio $\eta=0.20$ according to the bolometric luminosity given in Shen et al. (2008). Assuming a typical viscosity parameter of $\alpha=0.1$, this yields a lifetime of $t_{\text {disk }} \sim 1.4 \times 10^{8} \mathrm{yr}$ for the BLR. The BLR lifetime would be shorter if the Balmer line value of $v_{8}=4.1$ were used. Thus, we should expect coalescence to have taken place within the past 140 million years. This is likely comparable to the dynamical timescale for the host galaxy, so if this is indeed a recoiling black hole, follow-up observations might find evidence of a recent merger. The quasar-subtracted host galaxy fit would be consistent with a recent merger, but is insufficient to prove one has occurred. Alternatively, there might have been a long time delay between the merger and coalescence.

During these 140 million years, ignoring gravitational effects from the host galaxy, the ejected black hole could travel a distance of $d \sim v_{\text {ej }} t_{\text {disk }} \sim 175 \mathrm{kpc}$ for $v_{\text {ej }} \sim 1200 \mathrm{~km} \mathrm{~s}^{-1}$, or well outside of the host galaxy. Depending upon the mass of the host and the current separation, it is possible that SDSS 0956+5128 contains a recoiling black hole that will escape its host. Followup observations capable of resolving multiple point sources and determining both the distance to the center of the galaxy and mass of the host would be needed in order to determine its eventual fate.

Thus, the $\operatorname{Mg}$ II $(z \sim 0.707)$ line by itself, combined with the quasar narrow lines at $z \sim 0.714$ and $\mathrm{AO}$ observations of the host, presents a picture consistent with a post-recoil SMBH. However, the Balmer lines are entirely inconsistent with this explanation; they are better explained as a disk emitter. Perhaps the explanation is that SDSS $0956+5128$ is in some intermediate state in which a recoil has led to an eccentric disk, yet these two scenarios seem incompatible given the similar ionization potentials of $\mathrm{H} \beta$ and $\mathrm{Mg}$ II.

Might these scenarios be compatible? Because of the number of degrees of freedom involved in disk emitter models, it is possible to fit the Balmer lines of SDSS 0956+5128 as centered at the $z \sim 0.707$ redshift of $\mathrm{Mg}$ II rather than of the narrow lines, again with large $\varphi_{0}$, high eccentricity, and a large inner pericenter distance $\xi_{1}$. If the impulse delivered to the central black hole at coalescence is non-adiabatic, it may drive a circular accretion disk to become highly eccentric. There might then be some brief window in which Balmer emission is coming from an eccentric disk but $\mathrm{Mg}$ II is not yet. One test of this picture would be the C IV line profile, which cannot be investigated from the ground because it requires UV spectroscopy. Because $\mathrm{C}$ IV has an ionization potential placing it closer to the central black hole than $\mathrm{H} \beta$, it must also be double peaked. If such a model is plausible, though, it requires a combination of rare events: (1) a merger; (2) the correct spin and geometry for a large recoil; and (3) timing such that the quasar is observed in the brief time when the Balmer lines have become strongly asymmetric but the slightly higher-radius Mg II emission is not yet asymmetric. Since there are no known examples merely where (1) and (2) both occur, this scenario seems unlikely. However, this object appears to be unique among a catalog of $>10^{4}$ quasars containing both $\mathrm{H} \beta$ and $\mathrm{Mg}$ II, so it could be a rare glimpse of an object in transition between a circular and eccentric disk.

What is clear, however, is that SDSS $0956+5128$ is a challenge for current models of quasar accretion. If related to a postcoalescence black hole, it would be particularly compelling because it was identified from the spectrum alone, without the ability to resolve the host galaxy, since most mergers occur at too high a redshift for us to resolve the host. An ability to detect such sources from a large survey such as SDSS gives us the opportunity to better understand merger rates and galaxy evolution and to better calibrate our expectations for planned gravitational wave detectors. If SDSS $0956+5128$ is an exotic disk emitter, it will teach us something about the nature of quasar accretion, and in particular that $\mathrm{H} \beta$ and $\mathrm{Mg}$ II, despite similar ionization potentials, can arise from quite distinct regions. And, if SDSS $0956+5128$ is in a rare, post-merger transition state, a proper model may allow a better understanding of the quasar life cycle or duty cycle.

The authors thank Kevin Bundy, Masahiro Fukugita, Pat Hall, Daniel Proga, Robert Quimby, Allan Sadun, Masayuki Tanaka, and Alexander Tchekhovskoy for valuable comments. This work was supported by the World Premier International Research Center Initiative (WPI Initiative), MEXT, Japan. M.A.S., P.P., and R.A. acknowledge the support of NSF grant AST-0707266. C.L.S. and M.E. thank the Aspen Center for Physics and the NSF Grant No. 1066293 for hospitality during the editing of this paper.

\section{REFERENCES}

Abazajian, K., Adelman-McCarthy, J. K., Agüeros, M. A., et al. 2005, AJ, 129, 1755

Aihara, H., Allende Prieto, C., An, D., et al. 2011, ApJS, 193, 29

Ballo, L., Braito, V., Della Ceca, R., et al. 2004, ApJ, 600, 634

Barrows, R. S., Stern, D., Madsen, K., et al. 2012, ApJ, 744, 7

Bentz, M. C., Peterson, B. M., Netzer, H., Pogge, R. W., \& Vestergaard, M. 2009, ApJ, 697, 160

Bianchi, S., Chiaberge, M., Piconcelli, E., Guainazzi, M., \& Matt, G. 2008, MNRAS, 386, 105 
Bonning, E. W., Shields, G. A., \& Salviander, S. 2007, ApJ, 666, L13

Boroson, T. A., \& Lauer, T. R. 2009, Nature, 458, 53

Campanelli, M., Lousto, C. O., Zlochower, Y., \& Merritt, D. 2007, Phys. Rev. Lett., 98, 231102

Chen, K., \& Halpern, J. P. 1989, ApJ, 344, 115

Civano, F., Elvis, M., Lanzuisi, G., et al. 2010, ApJ, 717, 209

Civano, F., Elvis, M., Lanzuisi, G., et al. 2012, ApJ, 752, 49

Colpi, M., \& Dotti, M. 2009, arXiv e-prints

Comerford, J. M., Griffith, R. L., Gerke, B. F., et al. 2009, ApJ, 702, L82

Cushing, M. C., Vacca, W. D., \& Rayner, J. T. 2004, PASP, 116, 362

Decarli, R., Dotti, M., Montuori, C., Liimets, T., \& Ederoclite, A. 2010, ApJ, 720, L93

Di Matteo, T., Springel, V., \& Hernquist, L. 2005, in Growing Black Holes: Accretion in a Cosmological Context, ed. A. Merloni, S. Nayakshin, \& R. A. Sunyaev (Berlin: Springer), 340

Dotti, M., Colpi, M., \& Haardt, F. 2006, MNRAS, 367, 103

Eracleous, M., Livio, M., Halpern, J. P., \& Storchi-Bergmann, T. 1995, ApJ, 438,610

Escala, A., Larson, R. B., Coppi, P. S., \& Mardones, D. 2004, ApJ, 607, 765

Faber, S. M., Phillips, A. C., Kibrick, R. I., et al. 2003, Proc. SPIE, 4841, 1657 Häring, N., \& Rix, H.-W. 2004, ApJ, 604, L89

Hayano, Y., Takami, H., Guyon, O., et al. 2008, Proc. SPIE, 7015, 701510

Hayano, Y., Takami, H., Oya, S., et al. 2010, Proc. SPIE, 7736, 77360N

Herrmann, F., Hinder, I., Shoemaker, D. M., Laguna, P., \& Matzner, R. A. 2007, Phys. Rev. D, 76, 084032

Hoffman, L., \& Loeb, A. 2006, ApJ, 638, L75

Hudson, D. S., Reiprich, T. H., Clarke, T. E., \& Sarazin, C. L. 2006, A\&A, 453, 433

Jahnke, K., Elbaz, D., Pantin, E., et al. 2009, ApJ, 700, 1820

Kim, M., Ho, L. C., Peng, C. Y., \& Im, M. 2007, ApJ, 658, 107

Komossa, S., Burwitz, V., Hasinger, G., et al. 2003, ApJ, 582, L15

Komossa, S., Zhou, H., \& Lu, H. 2008, ApJ, 678, L81

Liu, X., Shen, Y., Strauss, M. A., \& Greene, J. E. 2010, ApJ, 708, 427

Loeb, A. 2007, Phys. Rev. Lett., 99, 041103

Luo, B., Brandt, W. N., Silverman, J. D., et al. 2009, ApJ, 695, 1227

Magain, P., Letawe, G., Courbin, F., et al. 2005, Nature, 437, 381

Maraston, C. 2005, MNRAS, 362, 799

Marconi, A., Axon, D. J., Maiolino, R., et al. 2009, ApJ, 698, L103

Martin, D. C., Fanson, J., Schiminovich, D., et al. 2005, ApJ, 619, L1

Marziani, P., Sulentic, J. W., Negrete, C. A., et al. 2010, MNRAS, 409, 1033
Max, C. E., Canalizo, G., \& de Vries, W. H. 2007, Science, 316, 1877

Mayer, L., Kazantzidis, S., Madau, P., et al. 2007, Multi-Scale Simulations of Merging Galaxies with Supermassive Black Holes (Berlin: Springer), 152

McGurk, R. C., Max, C. E., Rosario, D. J., et al. 2011, ApJ, 738, L2

McLure, R. J., \& Dunlop, J. S. 2004, MNRAS, 352, 1390

McLure, R. J., \& Jarvis, M. J. 2002, MNRAS, 337, 109

Merritt, D., \& Milosavljević, M. 2005, Living Rev. Relativ., 8, 8

Merritt, D., Storchi-Bergmann, T., Robinson, A., et al. 2006, MNRAS, 367 1746

Murray, N., Chiang, J., Grossman, S. A., \& Voit, G. M. 1995, ApJ, 451, 498

Onken, C. A., \& Kollmeier, J. A. 2008, ApJ, 689, L13

Peng, Y.-j., Lilly, S. J., Kovač, K., et al. 2010, ApJ, 721, 193

Peterson, B. 2008, An Introduction to Active Galactic Nuclei (Cambridge: Cambridge Univ. Press)

Pretorius, F. 2007, arXiv:0710.1338

Rafiee, A., \& Hall, P. B. 2011, MNRAS, 415, 2932

Rayner, J. T., Toomey, D. W., Onaka, P. M., et al. 2003, PASP, 115, 362

Richards, G. T., Fan, X., Newberg, H. J., et al. 2002, AJ, 123, 2945

Richards, G. T., Lacy, M., Storrie-Lombardi, L. J., et al. 2006, ApJS, 166, 470

Risaliti, G., Young, M., \& Elvis, M. 2009, ApJ, 700, L6

Rodriguez, C., Taylor, G. B., Zavala, R. T., et al. 2006, ApJ, 646, 49

Schnittman, J. D., \& Buonanno, A. 2007, ApJ, 662, L63

Shakura, N. I., \& Sunyaev, R. A. 1973, A\&A, 24, 337

Shen, Y., Greene, J. E., Strauss, M. A., Richards, G. T., \& Schneider, D. P. 2008, ApJ, 680, 169

Shields, G. A., Rosario, D. J., Smith, K. L., et al. 2009, ApJ, 707, 936

Skrutskie, M. F., Cutri, R. M., Stiening, R., et al. 2006, AJ, 131, 1163

Strateva, I. V. 2004, PhD thesis, Princeton Univ.

Strateva, I. V., Strauss, M. A., Hao, L., et al. 2003, AJ, 126, 1720

Tsalmantza, P., Decarli, R., Dotti, M., \& Hogg, D. W. 2011, ApJ, 738, 20

Vacca, W. D., Cushing, M. C., \& Rayner, J. T. 2003, PASP, 115, 389

Vanden Berk, D. E., Richards, G. T., Bauer, A., et al. 2001, AJ, 122, 549

Vestergaard, M. 2002, ApJ, 571, 733

Vestergaard, M., \& Peterson, B. M. 2006, ApJ, 641, 689

Wang, J., Dong, X., Wang, T., et al. 2009, ApJ, 707, 1334

Wilson, J. C., Henderson, C. P., Herter, T. L., et al. 2004, Proc. SPIE, 5492, 1295

Wright, E. L., Eisenhardt, P. R. M., Mainzer, A. K., et al. 2010, AJ, 140, 1868

Yanny, B., Rockosi, C., Newberg, H. J., et al. 2009, AJ, 137, 4377

Yu, Q., \& Tremaine, S. 2002, MNRAS, 335, 965 